\title{
COCONET: \\ A ROADMAP FOR CONTEXT-AWARE COOPERATION ENVIRONMENTS
}

\author{
Hans Schaffers \\ Telematica Instituut, hans.schaffers@telin.nl, THE NETHERLANDS
}

Amnon Ribak IBM Research Laboratory at Haifa, ribak@il.ibm.com, ISRAEL

Volker Tschammer FraunhoferFOKUS, tschammer@fokus.fhg.de, GERMANY

\begin{abstract}
This paper presents the approach and some results of the COCONET Roadmap project (IST-2001-37460). This project has as its objective to prepare a strategy and roadmap for RTD of high industrial impact in the area of context-aware collaborative environments for next generation business networks. The roadmap approach as presented here is based on scenarios for future work and business environments. Major challenges in future RTD are described and the approach is evaluated.
\end{abstract}

\section{INTRODUCTION}

The objective of this paper is to provide a systematic outlook to new forms of cooperation in work and business environments, their economic and societal drivers, and implications for research and technology development (RTD). This paper is based on findings of the COCONET Roadmap projecti. COCONET has developed a 5-10 year research agenda and roadmap of RTD challenges in the area of 'next generation context aware collaborative environments'. It was based on a process of scenario workshops and interactive roadmap development and validation, involving business and research stakeholders. Thus consensus was built with key players in identifying research themes for large-scale research and innovation directions.

The starting point of COCONET was the assessment of current weaknesses and opportunities in the European innovation system in IT-based work and business (European Commission, 2002). In e-work and e-business, Europe has strong RTD communities but there are difficulties in bringing them together. There is a need for more integrated approaches. There is also a lack of interoperable infrastructures. SMEs are found to be lagging behind the pace of developments. However, the new paradigm of networking and the European competence in tools for the support of managing and sharing knowledge are to be seen as European advantages. 
The Lisbon 2000 summit recognized the paradigm shift driven by globalisation and the new knowledge economy. A radical transformation of Europe's economy and society would be required. The European economy must be more dynamic if it is to match up with its major competitors. Among several other gaps and imbalances there is a skills gap, particularly notably in information technology. Taking this view further, the innovative potential of the future European economy will decisively rely on highly creative and flexible business and work environments, which are based on self-organisation, co-operation, and competence. Hierarchical structures will be more and more complemented or replaced by forms of networking. It seems probable that in a future unified Europe of one common market of labour and competencies national or cultural diversities will no longer be an obstacle for flexible and dynamic forms of work organisation. In contrast, facilities of future context-aware collaborative work environments must facilitate the exploitation of such cultural or national characteristics for the profit of the individual worker as well as for the co-operative undertakings she/he is involved in.

COCONET aims to contribute to this vision, focusing on scenarios of cooperative work environments that are person-centred and based on sharing of information, knowledge and applications. Supporting people to cooperate implies they are empowered to come together without any barriers to exploit business opportunities or to resolve problems. Cooperation environments also will be embedded in the collaborative business processes of networked organisations. They are context aware in their adaptiveness to situational characteristics such as task, performance, human needs, new participants, location, language, and computing resources.

In Chapter 2 we present the methodology of the roadmap development process. This process was highly scenario driven and resulted in 'maps' outlining the evolution of work environments, enabling technologies and research efforts required. In Chapter 3 we provide a selection of the important RTD challenges as identified.

\section{THE COCONET ROADMAP METHODOLOGY}

The COCONET roadmap is the result of a RTD strategy process. Important aspects of this process are the identification of trends, developments and challenges in society and business, technology, and markets and industries, and the analysis of the critical technologies and competencies shaping future markets and industries and responding to challenges. Additionally, the current state of the industry, technology and society, including its strengths and weaknesses, determines which future strategic paths are feasible and desirable. In this strategy and roadmap development process major industry and research partners were involved from the beginning. Based on development of scenarios for future cooperative work environments, requirements to ICTs were assessed and challenges in innovation and RTD identified over the time horizon. Departing from roadmap approaches as adopted by companies like Philips, our roadmap consists of four layers: the user (work and business) environment, the applications, the technology and competencies, and the RTD projects layers (Figure 1). For these layers, plausible milestones are projected over a time horizon of 10 years and interrelated to each other. 


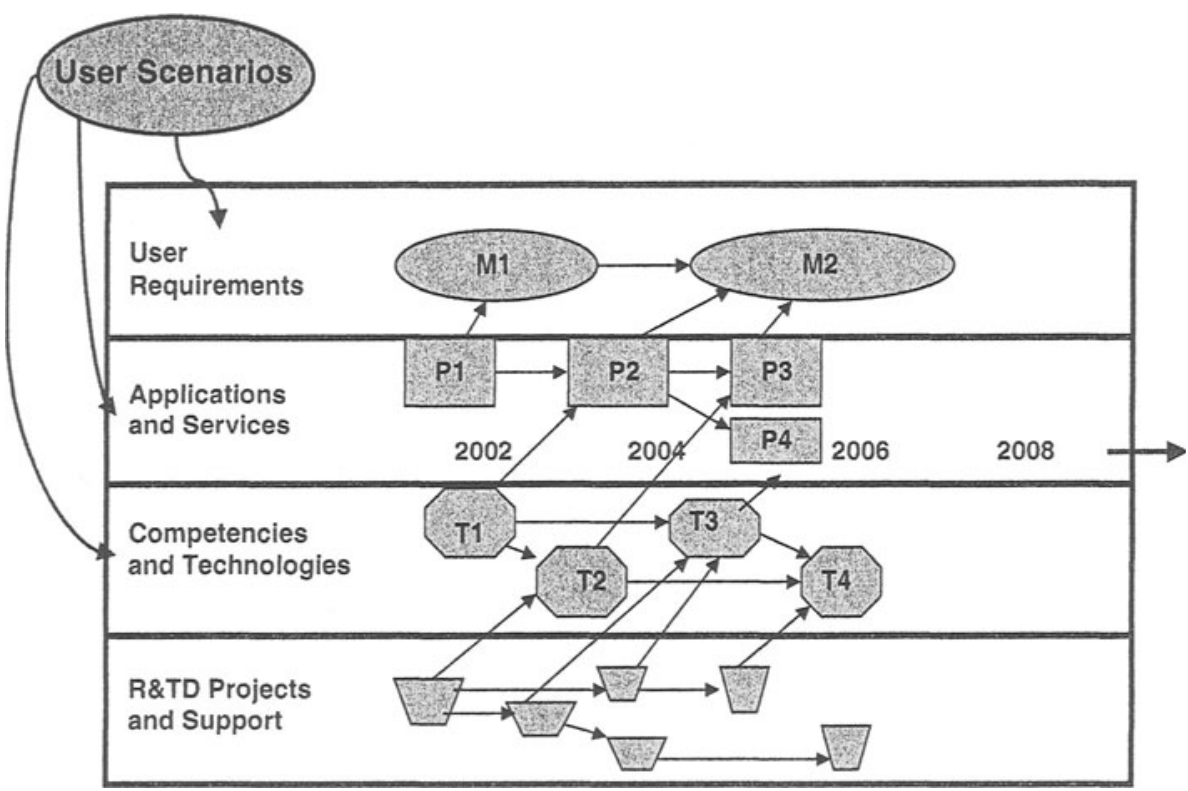

Figure 1: Structure of a scenario-driven roadmap

An analysis of bottlenecks and evolving needs in the work and business environment, where future ICTs could play a role to address them, was carried out. This was based on general sources of information as well as on the project's detailed experience in the media, chemical and manufacturing industries. For example in the chemical and manufacturing industries it is highly important to decrease the time between initial research, development of a product and bringing the product on the market. Additionally, it becomes critical to involve customers as early as possible in the design and testing process. New forms of cooperative support therefore integrate communication needs of employees, customers and producers, managing the product life cycle, and new ways of specialization and outsourcing are facilitated. The roadmap process was driven by development of visionary scenarios of context aware cooperative work environments. The scenarios were translated into human, organisational and business requirements as well as requirements to the enabling ICTs. These requirements then were mapped into competence areas - broad areas of knowledge and know-how - and technologies that constitute these areas. In the first stage of the scenario development process, four future human-oriented collaboration situations were conceived (Table 1). In the second stage the scenarios were revised and extended in order to bring them more in line with the focus of COCONET on context-aware human-oriented co-operative work. This was done through group discussions during COCONET workshops. As a result eight 'specific scenarios' have been developed: 1.Ad-hoc Collaboration; 2.Virtual /physical meeting; 3.Project Team Inception; 4.Multidisciplinary Management; 5.Mediation; 6.Transaction 
Processing; 7.Emergency Management; and 8.Distributed Management. Table 2 presents them in the two dimensions of time and task structure.

Table 1: Four scenarios of co-operative work and business

\begin{tabular}{|c|c|}
\hline $\begin{array}{l}\text { 1. "AD-HOC COLLABORATION" } \\
\text { Ad-hoc time-critical collaboration based } \\
\text { on individual collaboration needs } \\
\text { (person-to-person services) } \\
\text { - Ad-hoc communication based on } \\
\text { personalised communication devices, } \\
\text { supporting task switching } \\
\text { Includes emergency situations requiring } \\
\text { fast interaction / decision making }\end{array}$ & $\begin{array}{l}\text { 3. "TEAM INCEPTION AND LIFE CYCLE" } \\
\text { - Local collaboration; person-initiated } \\
\text { and supervised (local) supply chains / } \\
\text { transaction structures } \\
\text { - SME business integration } \\
\text { - Virtual meetings of chain actors to } \\
\text { negotiate, solve problems etc } \\
\text { - Chain actors involved in / able to } \\
\text { manage multiple supply chains }\end{array}$ \\
\hline $\begin{array}{l}\text { 2. "CO-OPERATIVE BUSINESS" } \\
\text { - Team building to respond or anticipate } \\
\text { on ad-hoc situations } \\
\text { Match the new human resource needs } \\
\text { /competence requirements to resources } \\
\text { available, solve conflict } \\
\text { - Supported by smart spaces, } \\
\text { personalised knowledge, intelligent } \\
\text { context-adaptable middleware, human- } \\
\text { computer interaction etc. }\end{array}$ & $\begin{array}{l}\text { 4. "LEARNING IN CO-OPERATIVE WORK" } \\
\text { Co-operative learning; highly diverse } \\
\text { highly flexible working group } \\
\text { - Empowering learners } \\
\text { - Virtual meeting space, synchronised } \\
\text { learning support } \\
\text { Individual learning in virtual } \\
\text { communities }\end{array}$ \\
\hline
\end{tabular}

Table 2: Detailed Scenarios

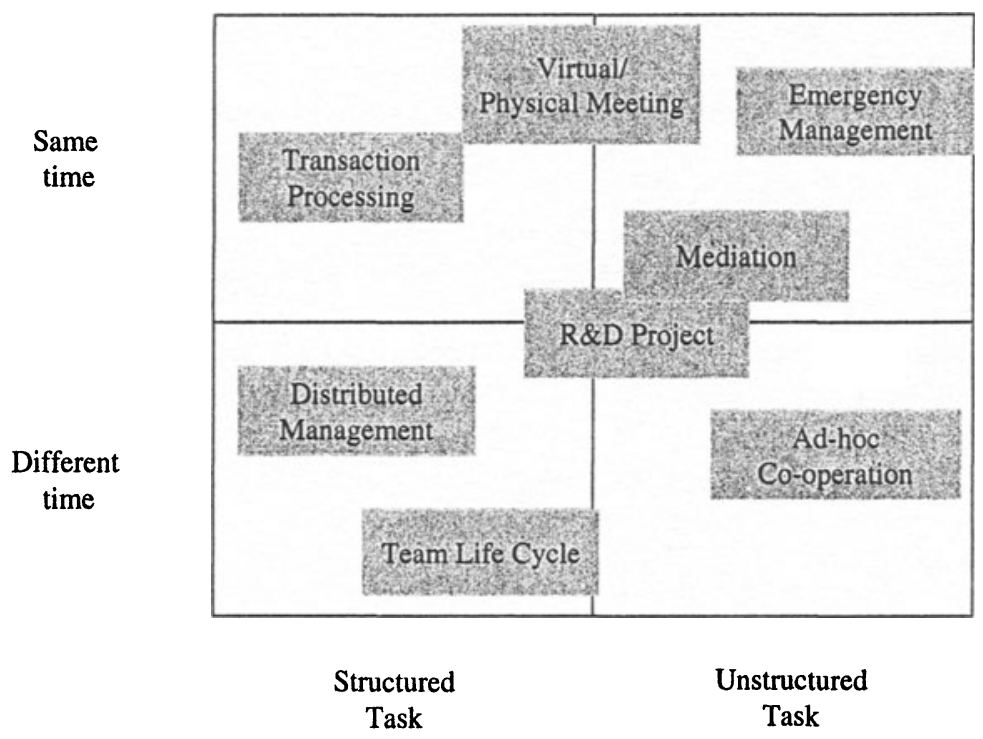


For each of these scenarios, innovations, RTD requirements and research challenges have been identified and explored, serving as inputs for the subsequent roadmap development process. The result of this process was that more than ten RTD areas could be identified in user environments, technological challenges and research projects and core areas could be distinguished from related areas (Figure 2) Some of the maps that have been developed in detail - using, in small groups, an interactive tool called the 'Roadmap Editor' - are presented below in Figure 3 and 4.

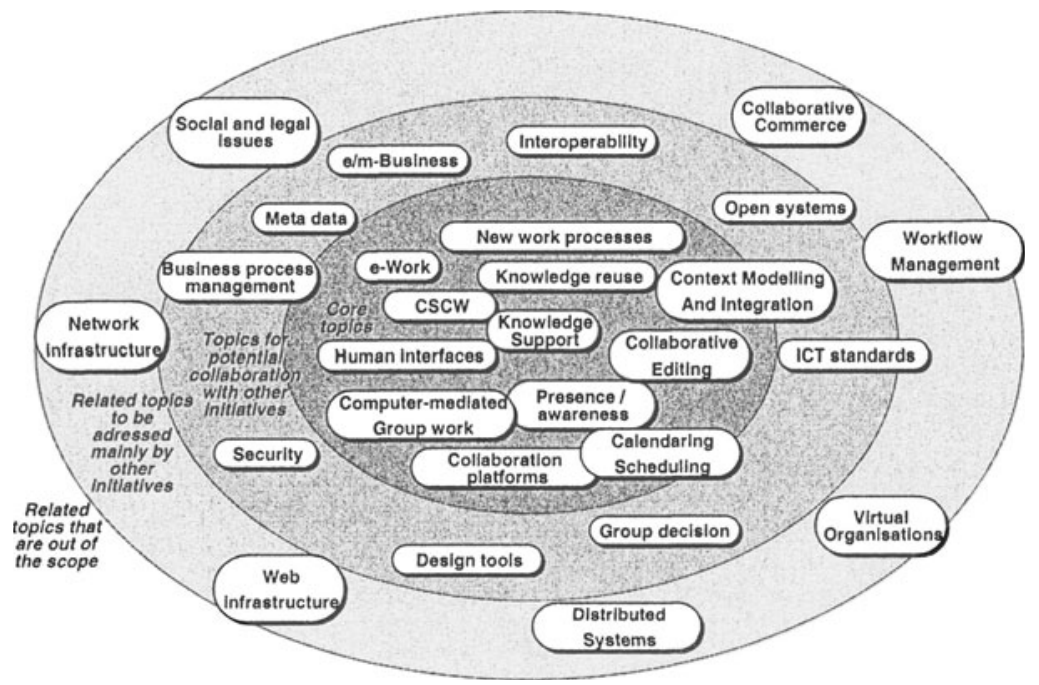

Figure 2: Core and related technology and competence areas

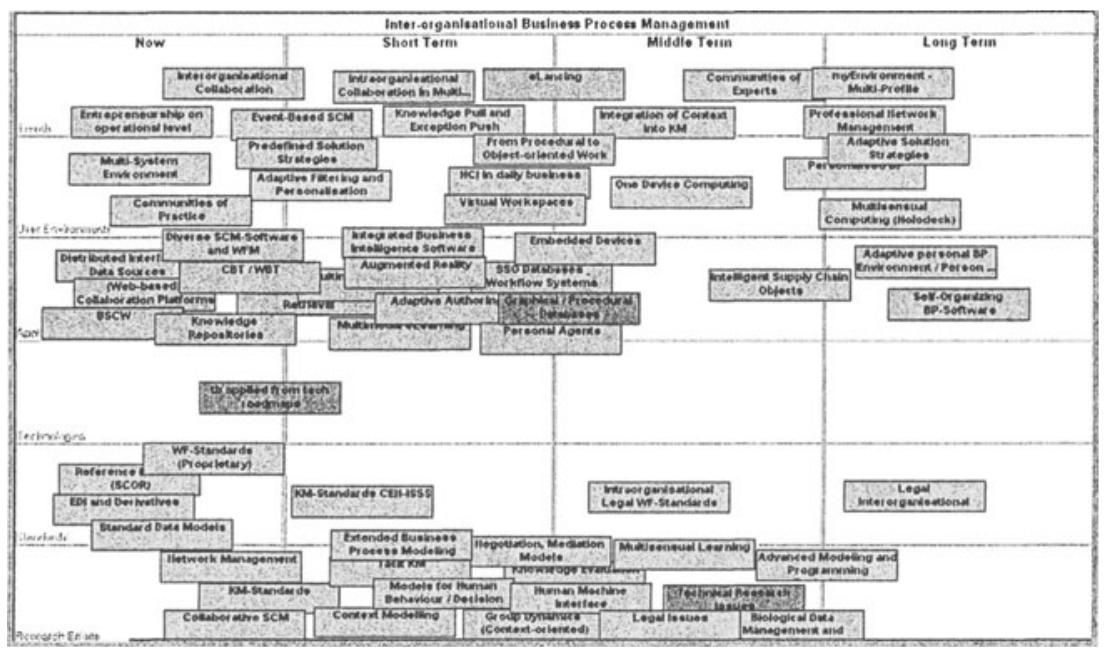

Figure 3: Roadmap example 1: Inter-organisational business process management 


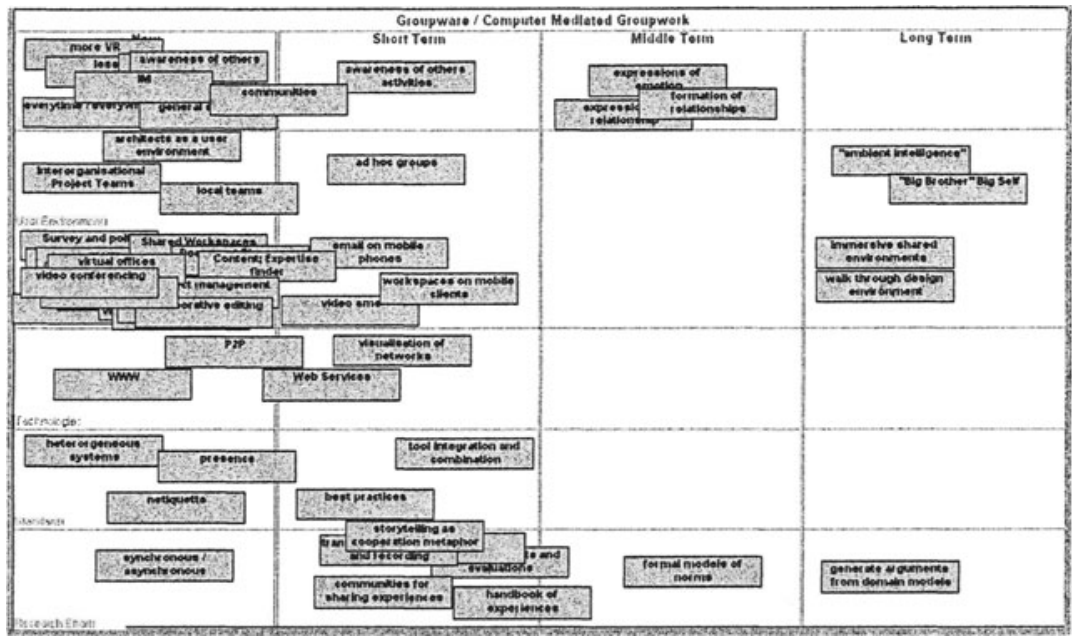

Figure 4: Roadmap example 2: Future co-operative workspaces

The Roadmap Editor tool provided a successful way to organize small-scale intensive group discussion in a structured way, and was an important step towards defining the final RTD challenges.

\section{CHALLENGES IN RTD AND INNOVATION}

Four major RTD challenges have been chosen for discussion in order to illustrate the roadmap results: 1.Human task support in networked environments; 2.Cooperation support embedded in networked enterprises; 3.Context-aware work environments; 4.Service-based cooperative workspaces. For more details see COCONET, 2003.

\subsection{Human task support in networked environments}

The employee in information-intensive, distributed, rapidly changing environments is solving ad-hoc tasks in emergency situations, is requiring access to and cooperation with competencies distributed across organisations, is navigating in shared and dynamic workspaces; is interacting in ambient environments. Therefore employees need adequate support when acting in different roles and in different domains. In this area we see challenges to provide human workers with presence and awareness support in dynamically changing environments, and to provide them with adequate means for human-computer interaction. Traditional groupware support takes as its point of departure the way task groups are working together. More and more there is a tendency towards communities of networked individuals, and to establish more informal and human oriented cooperative support. Another set of challenges is in providing users with more personalised and context-aware 
information resources. This is the area of information personalisation, which is also of importance for knowledge management and learning. Much emphasis is on location as context variable, but more dimensions of context should be investigated. In information personalisation, one area of work is in the use of profiles for filtering and structuring information. Other areas here are collaborative filtering, learning personalisation, the use of metadata and ontologies in finding and enriching content, as well as standardisation. A related direction is in realising presence and awareness support and instant messaging in workplace contexts. Such features provide continuous information about presence and availability of others. For mobile knowledge workers this technology promises not only to improve reachability and availability, but also to reduce the amount of inconvenient interruptions.

\subsection{Cooperation support embedded in networked enterprises}

Human work environments or workspaces are connected with business processes of networked organisations. Organisations wish to work together on the basis of business opportunities, or on the basis of emergent problems to solve. In the end it is the people in a work environment who start working together. One of the challenges in this field is to enable a workspace to connect with or to evolve from business processes of networked partners, like design, engineering, operations, logistics, administration and management. In this way the challenge is to enable networked business process integration, and such process integration in turn should support the co-operative work tasks to be implemented by collaborating organisations. In this area we see key research challenges in the seamless integration of virtual and physical workspaces; in providing integrated collaborative solutions, provided by collaborative service providers, supporting the complete project life cycles; in developing new workspace concepts for different and cross-sector domains and in supporting process integration.

\subsection{Context awareness in cooperative environments}

An initial issue is how to define and model contexts. A second issue is how to define services supporting context awareness. Context awareness can be approached from different sides: the modelling of context, the contextualisation of information (e.g. information personalisation, has been dealt with earlier), the development of context aware services, the development of systems enabling composition of cooperative systems according to context. As regards modelling and integration of context, it is important to cope with the issue of modelling contexts for representing knowledge and reasoning. Focus is on the real-world collaborative work situation where different types of context (e.g. private context of people and shared context of a project) and their interaction must be considered. Research challenge is contextbased representation of knowledge and reasoning, and building of 'procedural' context. Context modelling and integration, context acquisition, service definition and creation; services for presence and awareness support; personalisation in distributed group contexts will be challenges to represent in these application services. Increasingly it becomes important to focus on the way context information can be used in enriching the interactions between the user and the services. Context aware adaptive services may effectively use context information. Existing services may be combined to provide richer composite services assembled to meet user requirements. The research directions in this area are: Service composition to 
provide value added services; requiring the expression of flow of control and information between elemental services; drawing strongly on business process modelling and languages for enactable work flows; and service architectures that enable service discovery, composition and integration.

\subsection{Service-based cooperative workspaces}

Service integration between different organisations or business entities requires a loose coupling of highly heterogeneous systems over the Internet. Web services are based on Internet standards and fulfil the need for middleware allowing applications to collaborate irrespective of their concrete implementation. In adding semantic web support to web services, we are moving into the direction of a 'service web', and applications evolve towards application services enabling one-to-many software delivery and the construction of on-demand applications. One of the examples fitting perfectly in the COCONET vision is the modular and on-demand designed project office workspace. Applications more and more evolve to sets of interoperable eservices. These sets can be assembled and configured, on the fly, over the Internet to perform functions or support tasks or execute business processes. In this way functionalities such as instant messaging, conferencing, presence awareness, unified messaging, e-mail, virtual workspaces are integrated. This development is departing from the web services paradigm as a way to provide application functions available through the Internet on the basis of standard protocols. Areas of RTD in this field are new technologies and standards for service description, discovery, composition; and the integration of (real-time) cooperation functionalities in supply chain management and other business applications (XRM), for example presence and instant messaging in negotiating business transactions.

\section{FINAL REMARKS}

Roadmap development is part of a participatory priority setting process, thus it is more an art than a science. Our claims are based on inter-subjectivity. The combination of various approaches however has proven to be effective. A comparison among different roadmap approaches would be useful to derive learning experience and 'best practice'. In our view, roadmap exercises have to be consistent, plausible, relevant and challenging, confronting traditional frames of thinking. The proof of the pudding however is in the eating: whether the roadmap will become a meaningful instrument in policy and strategy discussions about future cooperative work and business environments including the setting of priorities for RTD.

\section{References}

1. COCONET, ' Research Agenda and Roadmap'. Deliverable D2.3. http://coconet.telin.nl/, June 2003.

2. European Commission, ISTAG Report 'Strategic Orientations and Priorities for IST in FP6', 2002.

\footnotetext{
i COCONET is funded by the European Commission (IST-2001-37460) and is completed June 2003. The authors acknowledge contributions of consortium partners and members of the Special Interest Group.
} 